\title{
Saberes Populares Sobre a Utilização de Recursos Naturais para Tratamento de Doenças na Infância em Barbalha, Ceará, Brasil
}

\section{Popular Knowledge about Use of Natural Resources for the Treatment of Childhood Diseases in Barbalha, Ceará, Brazil}

\author{
Izabel Cristina Santiago Lemos de Beltrão*a; Luanna Gomes da Silva ${ }^{\mathrm{a}}$; Jênifa Cavalcante dos Santos Santiago ${ }^{\text {b; }}$ Roseli
} Barbosa $^{\text {a }}$; Marta Regina Kenrtopf ${ }^{\mathrm{a}}$; George Pimentel Fernandes ${ }^{\mathrm{a}}$

\author{
${ }^{a}$ Universidade Regional do Cariri. CE, Brasil. \\ bUniversidade Federal do Ceará. CE, Brasil. \\ *E-mail: izabeldebeltrao@gmail.com
}

\begin{abstract}
Resumo
O uso de recursos naturais para tratar enfermidades infantis tem grande predominância. Assim, este estudo objetiva investigar os saberes de uma comunidade tradicional da zona rural do município de Barbalha, Nordeste do Brasil, sobre a utilização de recursos naturais para o tratamento de doenças prevalentes na infância. A pesquisa descritiva foi realizada com 54 participantes. Os dados foram coletados por questionário e entrevista semiestruturada e analisados por meio de estatística descritiva. Uma parcela significativa dos entrevistados $(50,0 \%)$ relatou utilizar frequentemente plantas e/ ou partes de animais para tratar e prevenir doenças $(\mathrm{p}<0,05)$. Ao comparar o conhecimento de homens e mulheres referente ao uso de recursos naturais, houve diferença estatística em relação ao número de espécies citadas por homens e por mulheres para as doenças diarreicas na infância $(\mathrm{p}<0,05)$. Comparado o conhecimento dos informantes mais velhos e mais jovens, houve associação significativa entre a idade e o número de espécies citadas quando considerada as infecções respiratórias agudas $(\mathrm{p}<0,05)$. Os relatos evidenciaram o uso de recursos naturais para tratar doenças na infância, incluindo homens e mulheres de diferentes grupos etários, o que reflete a perpetuação dessa prática. Os aspectos e práticas do saber popular podem ser um importante aliado na busca pela assistência integral à saúde da criança.
\end{abstract}

Palavras-chave: Medicina Tradicional. Plantas Medicinais. Saúde da Criança.

\begin{abstract}
The use of natural resources to treat childhood illnesses is prevalent. Thus, this study aims to investigate the knowledge about the use of natural resources for the treatment of childhood prevalent diseases in a traditional rural community in the city of Barbalha, northeastern Brazil. Descriptive research was carried out with 54 participants. Data were collected by questionnaire and semi-structured interview and analyzed using descriptive statistics. A significant number of respondents (50.0\%) reported frequently using plants and/or animal parts to treat and prevent disease $(p<0.05)$. When comparing the knowledge of men and women, there was a statistical difference regarding the number of species cited by men and women for childhood diarrheal diseases $(p<0.05)$. Comparing the knowledge of older and younger informants, there was a significant association between age and number of species cited when considering acute respiratory infections $(p<0.05)$. Reports highlighted the use of natural resources to treat childhood illnesses, including men and women of different age groups, reflecting the perpetuation of this practice. The aspects and practices of popular knowledge can be an important ally in the search for comprehensive child's health care.
\end{abstract}

Keywords: Traditional Medicine. Medicinal Plants. Child's health.

\section{Introdução}

A medicina tradicional (MT) se caracteriza como um sistema médico baseado em crenças culturais de determinados povos, ou seja, em práticas que atravessaram gerações e permanecem vivas até a atualidade (LANGDON, 2014; SILVA et al., 2017). Em sua abrangência, a MT envolve, entre outras práticas, o uso de plantas medicinais e de medicamentos confeccionados a partir de partes do corpo do animal, ou ainda de produtos de seu metabolismo, bem como de minerais, além dos rituais místicos e religiosos (ZENI et al., 2017).

Tendo em vista o cuidado à saúde, ao longo das últimas décadas, as práticas da MT estão sendo muito incentivadas nos sistemas de saúde, especialmente na Atenção Primária em Saúde (CONTATORE et al., 2015; SOUSA; TESSER, 2017). No Brasil, a regulamentação das práticas da MT está contida na Política Nacional de Práticas Integrativas e Complementares em Saúde (PNPICS) e, no caso do uso de plantas medicinais, pela Política Nacional de Plantas Medicinais e Fitoterápicos (BRASIL, 2008; BRASIL, 2015; SOUSA; HORTALE; BODSTEIN, 2018).

Nesse contexto, compreende-se que o uso de recursos naturais, tais como plantas e partes de animais, é vívido e dinâmico em diversas comunidades e integrado nos seus sistemas populares de cuidados à saúde, o que envolve, indubitavelmente, os cuidados diretos prestados às crianças por mães e familiares. Nesse aspecto, no que concerne ao contexto da saúde infantil no Brasil, entre os agravos predominantes na população de 0 a 5 anos se destacam as infecções respiratórias agudas (IRA), a diarreia e a anemia como os mais comuns (SAPAROLLI; ADAMI, 2007; SANTOS; QUINTAO; ALMEIDA, 2010).

Sendo assim, considerando a MT, Souza et al. (2011) revelam que o uso de plantas medicinais no tratamento de enfermidades infantis tem grande predominância, 
principalmente, na forma de chás. Além disso, Fischer, Palodeto e Santos (2018) evidenciam, em seu estudo, diversas partes dos animais utilizadas com fins medicinais, tendo destaque o uso dos tecidos internos, predominantemente o adiposo. Por isso, torna-se relevante o conhecimento dos aspectos pertinentes ao uso de recursos naturais, como plantas e/ou animais, principalmente, por parte dos profissionais de saúde, no intuito de orientar os familiares para a utilização de tal prática de maneira segura e eficaz, respeitando, contudo, os aspectos culturais e de crenças inerentes à comunidade.

Portanto, o presente estudo tem por objetivo investigar o conhecimento de uma comunidade tradicional da zona rural do município de Barbalha, na Região do Cariri cearense, sobre a utilização de recursos naturais para o tratamento de doenças prevalentes na infância.

\section{Material e Métodos}

Trata-se de um estudo de natureza descritiva, com abordagem quantitativa, realizado na comunidade Santo Antônio, localizada na zona rural do município de Barbalha, na Região do Cariri cearense, Brasil.

Barbalha é um município situado a 553 quilômetros da capital do Ceará, Fortaleza. Ainda compõe o triângulo do Crajubar em conjunto com as cidades de Crato e do Juazeiro, que exerce importante atuação socioeconômica para a Região do Cariri. Em Barbalha é possível localizar diversas comunidades, entre essas a comunidade do Santo Antônio que foi escolhida como local do estudo por se enquadrar como uma comunidade tradicional (HAVERROTH, 2013), sendo reconhecida regionalmente por ser culturalmente diferenciada, tendo a tradição oral como o principal meio de difusão de seus costumes, em especial, aqueles relacionados à Medicina Popular.

Os participantes envolvidos na pesquisa foram indivíduos residentes da comunidade Santo Antônio. A seleção da amostra ocorreu a partir da tática de SnowBall (bola de neve). Tal método se caracteriza como não probabilístico, uma vez que não possibilita uma determinação absoluta da quantidade de integrantes. Em geral, a triagem da população ocorre a partir de uma cadeia da seguinte maneira: uma pessoa é indicada por um informante-chave que, por sua vez, indicará outra e, assim, sucessivamente. A repetição de um ou mais participante representa a saturação amostral (BALDIN; MUNHOZ, 2011; VINUTO, 2014). Assim, para a construção da amostragem se fez necessário, primeiramente, um contato inicial com a comunidade com as finalidades de autorização pelo líder comunitário para desenvolver a pesquisa, bem como para se estabelecer um vínculo de confiança, isto é, um "rapport" (ALBUQUERQUE; LUCENA; CUNHA, 2010). E, sendo assim, um total de 54 informantes participaram da coleta de dados.

O instrumental utilizado foi composto por: questionário fechado para caracterização socioeconômica dos sujeitos da pesquisa; roteiro de entrevista semiestruturada e gravador de áudio para obtenção das respostas. A análise dos dados foi constituída por meio da estatística descritiva (frequência simples e percentual) e o teste de hipóteses, através de técnicas não paramétricas descritivas e de inferencial, que propicia a comparação do conhecimento referente ao uso de recursos naturais para o tratamento de doenças prevalentes na infância expresso por pessoas do sexo feminino e por pessoas do sexo masculino.

Essa comparação também ocorreu considerando os indivíduos de diferentes idades, por distribuição de grupos etários (12 - 21 anos; $22-29$ anos; $30-45$ anos; 46 - 59 anos; 60 - 75 anos; 76 - 90 anos). Isso se tornou possível mediante o levantamento do número de espécies citadas por cada indivíduo para cada agravo considerado, a saber: infecções respiratórias agudas; diarreias e anemia.

Para interpor o conhecimento de pessoas do sexo feminino e do sexo masculino, e de diferentes grupos etários, com a finalidade de estabelecer parâmetros de comparação, foi utilizado o teste de hipótese não paramétrico Exato de Fisher ou, como é mais comumente conhecido, o teste do qui-quadrado $\left(x^{2}\right)$. Além do teste do $x^{2}$, foi empregado o Teste da Razão de Verossimilhança, que permite a estimação dos parâmetros de modelos e a realização de testes de hipóteses relativos às restrições lineares e não lineares ao vetor de parâmetros estabelecidos (PIMENTEL-GOMES, 1990). A verificação de tais dados foi mediada pelo uso do software Statistical Package for the Social Sciences - pacote estatístico para as Ciências Sociais, versão 13.0, considerando-se o nível de significância aplicado de 0,05 .

Cabe ressaltar que o transcorrer da pesquisa atendeu a todas as Exigências das Diretrizes e Normas da Pesquisa Envolvendo Seres Humanos, regulamentada pela Resolução 466/12 do Conselho Nacional de Saúde, assim como foi implementada após a aprovação do Comitê de Ética em Pesquisa (CEP) sob o parecer $n^{\circ} 705.497$.

\section{Resultados e Discussão}

O perfil dos informantes que participaram da pesquisa está detalhado no Quadro 1.

Quadro 1 - Perfil dos informantes do Sítio Sto. Antônio, Barbalha (CE), Brasil

\begin{tabular}{|l|l|c|c|}
\hline Município & Localidade & N & \% \\
\hline Barbalha - CE & $\begin{array}{l}\text { Sítio Sto. } \\
\text { Antônio }\end{array}$ & 54 & 100 \\
\hline Sexo & $\mathbf{N}$ & $\mathbf{\%}$ \\
\hline Masculino & 24 & 44,44 \\
\hline Feminino & 30 & 55,56 \\
\hline Faixa Etária & $\mathbf{N}$ & $\mathbf{\%}$ \\
\hline $12-21$ & 9 & 16,67 \\
\hline $22-29$ & 11 & 20,37 \\
\hline $30-45$ & 15 & 27,78 \\
\hline
\end{tabular}




\begin{tabular}{|c|c|c|}
\hline $46-59$ & 11 & 20,37 \\
\hline $60-75$ & 3 & 5,56 \\
\hline $76-96$ & 5 & 9,25 \\
\hline Tempo de Residência na Área & $\mathbf{N}$ & $\%$ \\
\hline$<5$ anos & -- & -- \\
\hline$\geq 5<10$ anos & 3 & 5,56 \\
\hline$\geq 10<20$ anos & 8 & 14,81 \\
\hline$\geq 20<30$ anos & 12 & 22,22 \\
\hline$\geq 30<40$ anos & 8 & 14,81 \\
\hline$\geq 40<50$ anos & 11 & 20,37 \\
\hline$\geq 50<60$ anos & 9 & 16,67 \\
\hline$\geq 60$ & 3 & 5,56 \\
\hline \begin{tabular}{|l|} 
Escolaridade \\
\end{tabular} & $\mathbf{N}$ & $\%$ \\
\hline Não-Escolarizado & -- & -- \\
\hline Ensino Fundamental Incompleto & 15 & 27,78 \\
\hline Ensino Fundamental Completo & 3 & 5,56 \\
\hline Ensino Médio Incompleto & 12 & 22,22 \\
\hline Ensino Médio Completo & 23 & 42,59 \\
\hline Outros & 1 & 1,85 \\
\hline Ocupação & $\mathbf{N}$ & $\%$ \\
\hline Agente comunitário de saúde & 1 & 1,85 \\
\hline Agricultor(a) & 28 & 51,85 \\
\hline Aposentado & 9 & 16,67 \\
\hline Autônomo & 3 & 5,56 \\
\hline Comerciário & 1 & 1,85 \\
\hline Estudante & 6 & 11,11 \\
\hline Manicure & 1 & 1,85 \\
\hline Pedreiro & 1 & 1,85 \\
\hline Professor & 1 & 1,85 \\
\hline Vendedor & 3 & 5,56 \\
\hline Estado Civil & $\mathbf{N}$ & $\%$ \\
\hline Solteiro (a) & 13 & 24,07 \\
\hline Casado (a) & 38 & 70,37 \\
\hline Viúvo (a) & 3 & 5,56 \\
\hline Divorciado (a) & -- & -- \\
\hline
\end{tabular}

Fonte: Dados da pesquisa.

No que refere à porcentagem quanto ao sexo, os resultados se assemelham com os do estudo de Zucchi et al. (2013), em que houve uma amostra equilibrada no que diz respeito ao número de informantes do sexo feminino (54\%) e do sexo masculino (46\%), afirmando em seus resultados que, na localidade em que ocorreu a investigação, o uso de plantas para fins medicinais é independente do sexo e da faixa etária.

Quanto aos grupos etários, os que tiveram maior representatividade foram aqueles em que os participantes apresentaram: 30 a 45 anos (27,78\%), 22 a 29 anos (20,37\%) e 46 a 59 anos $(20,37 \%)$. Nesse aspecto se destaca que alguns estudos brasileiros, que abrangem a temática de cuidado de saúde, no contexto da medicina tradicional, não costumam direcionar suas amostras para informantes que estejam na faixa etária em que se compreende a adolescência e os adultos jovens, dando primazia para idosos e adultos maduros (LIMA et al., 2011; FEIJÓ et al., 2013).

Segundo Heisler et al. (2012), parte-se do pressuposto de que o saber relacionado ao uso de recursos naturais no cuidado à saúde é dominado preponderantemente por pessoas idosas, classificadas como as detentoras de um maior conhecimento, fomentado no decorrer de sua própria vivência, acerca de práticas alternativas de cuidado.

Esse fator, contudo, prejudica a comparação e implicações referentes à preservação do saber tradicional em diferentes gerações, e de que formas os mais jovens compreendem, utilizam e perpetuam o saber transmitido pelos mais velhos.

Em relação ao nível de escolaridade $42,59 \%$ dos informantes relataram possuir Ensino Médio completo. Enquanto 27,78\% dos entrevistados professavam ter o Ensino Fundamental incompleto. Na pesquisa desenvolvida por Souza et al. (2013), realizada na Região Nordeste, também houve predomínio daqueles que cursaram o Ensino Fundamental incompleto $(40,9 \%)$.

\subsection{Conhecimento tradicional: uso e fontes de saber}

O gráfico da Figura 1 expressa a frequência com que os entrevistados utilizam plantas e/ou animais para fins de tratamento de doenças.

Figura 1 - Frequência com que os informantes da comunidade Sto. Antônio (Barbalha - CE) relataram utilizar plantas e/ou animais para o tratamento e a prevenção de doenças

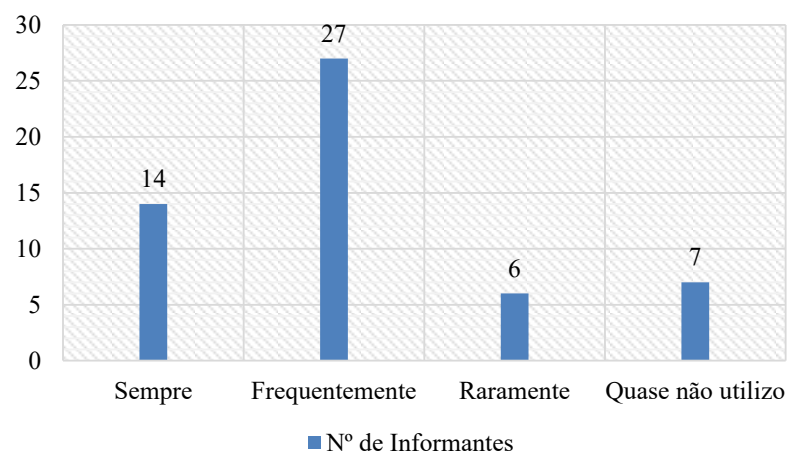

Fonte: Dados da pesquisa.

Desse modo, observa-se que $25,93 \%$ dos informantes relataram "sempre" utilizar plantas, 50\% referiram uso "frequente" das plantas, $11,11 \%$ apontaram utilizar "raramente", enquanto $12,96 \%$ dos participantes afirmaram quase não utilizar. Portanto, traçando um comparativo com os dados apresentados no gráfico da Figura 1, na pesquisa realizada por Feijó et al. (2013), em Ilhéus (Bahia - BA) $97,7 \%$ dos entrevistados afirmaram fazer uso regular de plantas medicinais. Nesse aspecto, Giraldi e Hanazaki (2010) mencionam que a utilização dos recursos naturais no contexto dos cuidados à saúde é um mecanismo de reforço das práticas tradicionais, bem como possibilita um maior contato e interação humana com elementos diversos da biodiversidade.

No que diz respeito aos tipos e às formas de transmissão de conhecimentos instituídos referentes ao uso de plantas e de 
animais para o tratamento de doenças comuns na infância, foi relatado que as pessoas residentes na comunidade do estudo aprenderam a utilizar esses recursos naturais, especialmente, por meio da tradição oral, mediada por familiares. Assim, os participantes da pesquisa citaram os familiares por 76 vezes, com destaque para as mães, mencionadas 32 vezes, as avós (16 vezes) e os pais (15 vezes).

Badke et al. (2012, p.363) "constataram que o aprendizado do uso e manipulação de plantas medicinais têm sua origem no contexto familiar", ressaltando que a maioria dos entrevistados teve contato com o uso de plantas medicinais na infância, ao observar o uso dessa prática por mães e avós.

Este padrão voltado para a transmissão oral do conhecimento tradicional e a predominância de mulheres sobre os saberes referentes ao uso de recursos naturais é evidente em outros estudos publicados em território nacional (FREITAS et al., 2012; MARCHESE et al., 2009). $\mathrm{O}$ que demonstra um certo protagonismo feminino no que concerne à utilização de práticas alternativas relacionadas aos cuidados com à saúde dos demais membros da família e da comunidade.

3.2 Conhecimento referente à utilização de plantas e/ ou de animais entre indivíduos do sexo feminino e entre indivíduos do sexo masculino

Com a finalidade de comparar o conhecimento esboçado durante as entrevistas entre homens e mulheres, no que tange ao uso de recursos naturais para o tratamento de doenças prevalentes na infância, foram empregados testes de hipóteses (Quadro 2).

Quadro 2 - Associação entre número de espécies citadas pelos informantes da comunidade Sto. Antônio (Barbalha - CE) e sexo

\begin{tabular}{|c|c|c|c|}
\hline & \multicolumn{2}{|c|}{ Sexo } & \multirow[b]{2}{*}{ p-valor } \\
\hline & Masculino & Feminino & \\
\hline IRA & $f(\%)$ & $f(\%)$ & \multirow{5}{*}{$0,060^{*}$} \\
\hline$<=2$ & $12(50,0)$ & $8(26,7)$ & \\
\hline 3 a 5 & $10(41,7)$ & $12(40,0)$ & \\
\hline 6 a 8 & $2(8,3)$ & $7(23,3)$ & \\
\hline$>=9$ & $0(0,0)$ & $3(10,0)$ & \\
\hline \multicolumn{4}{|c|}{ Diarreia } \\
\hline$<=2$ & $17(70,8)$ & $13(43,3)$ & \multirow{3}{*}{$0,041 *$} \\
\hline 3 a 5 & $7(29,2)$ & $14(46,7)$ & \\
\hline 6 a 8 & $0(0,0)$ & $3(10,0)$ & \\
\hline \multicolumn{4}{|c|}{ Anemia } \\
\hline$<=2$ & $22(91,7)$ & $24(80,0)$ & \multirow{2}{*}{$0,230 * *$} \\
\hline 3 a 5 & $2(8,3)$ & $6(20,0)$ & \\
\hline
\end{tabular}

IRA - Infecção respiratória aguda. *razão de verossimilhança; **teste $\mathrm{x}^{2}$. Fonte: Dados da pesquisa.

O teste do $x^{2}$ foi usado quando as frequências observadas foram maiores que 5. Em contrapartida, a razão de verossimilhança foi utilizada quando as frequências observadas foram menores que cinco.

Os resultados expressos no Quadro 2 demonstram que existe diferença estatística em relação ao número de espécies citadas por homens e por mulheres para as doenças diarreicas na infância $(\mathrm{p}<0,05)$.

Quando se considera o fator sexo, alguns pesquisadores afirmam que as mulheres são as detentoras de mais conhecimento tradicional, embora seja essa uma afirmação genérica, as justificativas, muitas vezes, repousam em questões culturais, relacionadas ao cuidado desempenhado pela mulher para com os demais membros da família, não sendo, portanto, infundadas (LIMA et al., 2011; FERREIRA; LOURENÇO; BALIZA, 2014).

Inclusive, pesquisas como de Oliveira, Barros e Moita Neto (2010), em que informantes do sexo masculino superaram informantes do sexo feminino não são comuns. Isso ocorre, muitas vezes, em função de condições específicas observadas no local da coleta de dados, tais como características e situações singulares da comunidade tradicional considerada e objeto do estudo em questão.

$\mathrm{Na}$ pesquisa desenvolvida, indivíduos de ambos os sexos revelaram conhecimentos diversos acerca do uso de espécies para finalidades terapêuticas relacionadas à saúde da criança, exceto quando considerada a doença "diarreia", na qual o conhecimento de indivíduos do sexo feminino foi sobressalente.

Esses achados corroboram com os resultados de outros levantamentos etnobotânicos. Por exemplo, no estudo de Rodrigues e Andrade (2014), homens e mulheres integraram o grupo de $5 \%$ daqueles que citaram mais de 30 espécies para uso medicinal.

\subsection{Conhecimento referente à utilização de plantas e/ ou de animais entre indivíduos de diferentes grupos etários}

Com objetivo de comparar o conhecimento sobre o uso de plantas e/ ou de animais para o tratamento das doenças prevalentes na infância entre pessoas de diferentes faixas etárias foi utilizado o teste do e a razão de verossimilhança, conforme expresso na tabela 3.

Os participantes da pesquisa foram distribuídos em 6 grupos etários distintos: 12-21 anos; 22-29 anos; 30-45 anos; 46-59 anos; 60-75 anos e 76-90 anos, a partir de então, foi realizada a associação entre o número de espécies diferentes citadas por cada indivíduo para um dado agravo (IRA; diarreia ou anemia) e sua idade.

Considerando os grupos etários distintos, só houve associação significativa entre a idade e o número de espécies citadas quando considerada as IRA, em que se tem $\mathrm{P}<0,05$. Para as doenças diarreicas e anemia não houve relevância estatística, sendo $\mathrm{p}=0,053$ e $\mathrm{p}=0,068$, respectivamente. 
Quadro 3 - Associação entre número de espécies citadas pelos informantes da comunidade Sto. Antônio (Barbalha - CE) e faixa etária

\begin{tabular}{|c|c|c|c|c|c|c|c|}
\hline & \multicolumn{6}{|c|}{ Faixa Etária (em anos) } & \multirow[b]{2}{*}{ p-valor } \\
\hline & $12-21$ & $22-29$ & $30-45$ & $46-59$ & $60-75$ & $76-90$ & \\
\hline IRA & $f(\%)$ & $f(\%)$ & $f(\%)$ & $f(\%)$ & $f(\%)$ & $f(\%)$ & \multirow{5}{*}{$0,000^{*}$} \\
\hline$<=2$ & $8(88,9)$ & $7(63,6)$ & $0(0,0)$ & $3(27,3)$ & $0(0,0)$ & $2(40,0)$ & \\
\hline 3 a 5 & $1(11,1)$ & $4(36,4)$ & $11(73,3)$ & $2(18,2)$ & $2(66,7)$ & $2(40,0)$ & \\
\hline 6 a 8 & $0(0,0)$ & $0(0,0)$ & $4(26,7)$ & $3(27,3)$ & $133,3)$ & $1(20,0)$ & \\
\hline$>=9$ & $0(0,0)$ & $0(0,0)$ & $0(0,0)$ & $3(27,3)$ & $0(0,0)$ & $0(0,0)$ & \\
\hline \multicolumn{8}{|c|}{ Diarreias } \\
\hline$<=2$ & $8(88,9)$ & $9(81,8)$ & $7(46,7)$ & $4(36,4)$ & $0(0,0)$ & $2(40,0)$ & \multirow{3}{*}{$0,053^{*}$} \\
\hline 3 a 5 & $1(11,1)$ & $2(18,2)$ & $7(46,7)$ & $6(54,5)$ & $3(100,0)$ & $2(40,0)$ & \\
\hline 6 a 8 & $0(0,0)$ & $0(0,0)$ & $1(6,7)$ & $1(9,1)$ & $0(0,0)$ & $1(20,0)$ & \\
\hline \multicolumn{8}{|c|}{ Anemias } \\
\hline$<=2$ & $9(100,0)$ & $11(100,0)$ & $13(86,7)$ & $7(63,6)$ & $2(66,7)$ & $4(80,0)$ & \multirow{2}{*}{$0,068 * *$} \\
\hline 3 a 5 & $0(0,0)$ & $0(0,0)$ & $2(13,3)$ & $4(36,4)$ & $1(33,3)$ & $1(20,0)$ & \\
\hline
\end{tabular}

IRA - Infecção respiratória aguda. *razão de verossimilhança; **teste $\mathrm{x}^{2}$.

Fonte: Dados da pesquisa.

Provavelmente, esse resultado se justifica pelo número reduzido de espécies indicadas para diarreia e, em especial, anemia, quando comparadas com as IRA. Esse fator também foi observado em outros estudos, nos quais houve preponderância e maior concordância de indicações para sintomatologias relacionadas ao trato respiratório em comparação a outros grupos de patologias (AGUIAR; BARROS, 2012; CHAVES; BARROS, 2012; RODRIGUES; ANDRADE, 2014).

Considerando ainda os resultados para IRA, ao despeito do que se era esperado, as faixas etárias que abrangem os idosos não esboçaram representação no grupo de citação que envolvia um número igual ou superior a nove espécies diferentes, ficando concentrados, preponderantemente, no grupo de citação que compreende de 3-5 espécies diferentes. Um fator interessante que também foi observado na comunidade é que os idosos do grupo etário mais jovem citaram mais espécies que os idosos do grupo etário com idade mais avançada.

Nesse aspecto, acredita-se que esse resultado foi possível, não como um reflexo do saber tradicional apreendido durante toda a vida dos indivíduos, mas como decorrente de alterações fisiológicas relacionadas ao envelhecimento e às doenças crônico-degenerativas (CHARCHAT-FICHMAN, 2005).

\subsection{Perspectivas para o tratamento das Infeç̧ões Respiratórias Agudas, Diarreias e Anemia no contexto da saúde pública brasileira}

Nos Estados Unidos, um estudo evidenciou que 36\% das crianças faz uso de algum tipo de tratamento não convencional, sendo o uso da medicina herbária o mais comum. No Brasil, $25 \%$ dos produtos farmacêuticos vendidos a partir do ano de 1996 até 2001 eram derivados de produtos naturais, sendo inclusive a utilização de plantas medicinais para o manejo dos sintomas de doenças respiratórias, várias vezes, encarado como tratamento de primeira linha (LOPES et al., 2014).

No âmbito da saúde pública brasileira, especificamente considerando o SUS, a Política Nacional de Práticas Integrativas e Complementares (PNPIC) colabora com o incentivo para a inclusão e o uso racional de recursos naturais no tratamento de diversas patologias. Assim, o incentivo ao uso desses recursos naturais, inclusive, para uma abordagem sintomatológica das IRA, vem apresentando um crescimento vertiginoso nas duas últimas décadas (BRASIL, 2015).

Nesse aspecto, focando a estratégia de Atenção Integral às Doenças Prevalentes na Infância (AIDPI), pode-se observar essa tendência nos manuais elaborados pelo Ministério da Saúde. Por exemplo, nos casos relacionados as IRA, o manual, no módulo 4, orienta: "Convença-a (a mãe/responsável da criança) da importância de usar medidas caseiras em lugar dos medicamentos convencionais. Seja claro em sua explicação" (BRASIL, 2003, p.20).

Até mesmo quando a criança apresenta o quadro diagnóstico de pneumonia, os profissionais de saúde são incentivados a estimular o uso das chamadas "medidas caseiras", que podem ser, além do aumento da oferta de líquidos, no caso específico das IRA: mel de abelha ou outras medidas culturalmente aceitas (BRASIL, 2003).

Nos casos de diarreia, o tratamento domiciliar não incentiva o uso de medidas caseiras tais como chás, mas orienta que as mães/responsáveis das crianças administrem o soro de reposição oral (SRO), além de otimizar a oferta de líquidos, preconizando o soro de preparo caseiro e a água potável, além de incentivar o uso da "água de arroz" e de "caldos" (BRASIL, 2003).

Para o manejo da anemia, decorrente de deficiências nutricionais, a estratégia foca, principalmente, na administração de suplementos de ferro orais, bem como o uso do mebendazol. Contudo, os profissionais de saúde são incentivados a avaliar a alimentação da criança e a realizar um levantamento dos alimentos, que são acessíveis à família (BRASIL, 2003).

Vale destacar ainda que, no manual AIDPI, há o estímulo da oferta diária de carne e de vísceras para crianças com anemia, pois esses possuem ferro de melhor valor biológico. Entre os alimentos citados estão a beterraba, o fígado e o feijão 
(BRASIL, 2003). Com a gradual implementação da PNPCI, e a publicação de novos estudos, acredita-se que a tendência é que essas vertentes culturais passem a ser mais valorizadas por profissionais de saúde na prática assistencial, em especial, na atenção primária (BARROS, 2006).

Os relatos deste estudo evidenciaram o uso de recursos naturais para tratar doenças na infância, incluindo homens e mulheres de diferentes grupos etários, o que reflete a perpetuação dessa prática. Os aspectos e as práticas do saber popular podem ser um importante aliado na busca pela assistência integral à saúde da criança. Quando esses aspectos culturais se tornam desconhecidos ou negligenciados pelos profissionais de saúde, durante as consultas ou visitas domiciliares, esses podem se configurar em uma barreira intransponível, que prejudica a adesão ao plano terapêutico e, consequentemente, as condições gerais de saúde e restabelecimento da criança.

\section{Conclusão}

Os achados deste estudo enfatizam que a utilização de plantas e/ou partes de animais para o tratamento e prevenção de doenças prevalentes na infância é uma prática recorrente na comunidade tradicional em estudo, difundida principalmente pela tradição oral, mediada por familiares. Identificou-se também a difusão de saberes da MT entre homens e mulheres de diferentes faixas etárias, mas ainda a sobressalência do conhecimento de mulheres sobre recursos naturais para tratar doenças diarreicas e de idosos relativo ao uso de espécies diferentes para o tratamento de IRA.

Vale destacar que a pesquisa se limitou a abordagem de apenas uma comunidade tradicional. Todavia, considerando a abrangência do tema pesquisado, fazem-se necessárias novas pesquisas, que abordem indivíduos inseridos em outras comunidades tradicionais e, em diferentes contextos, que identifiquem outras variáveis capazes de infuenciar as práticas da medicina tradicional no cuidado da criança e de que forma o profissional de saúde pode abordar tais práticas no seu cenário de atuação.

\section{Referências}

AGUIAR, L.C.G.G.; BARROS, R.F.M. Plantas medicinais cultivadas em quintais de comunidades rurais no domínio do cerrado piauiense. Rev. Bras. Plantas. Med., v.14, n.3, p.419-434, 2012. doi: 10.1590/S1516-05722012000300001

ALBUQUERQUE, U.P.; LUCENA, R.F.P.; CUNHA, L.V.F.C. Métodos e técnicas na pesquisa Etnobiológica e Etnoecológica. Recife: Nupeea, 2010.

BADKE, M.R. et al. Saberes e práticas populares de cuidado em saúde com o uso de plantas medicinais. Texto Contexto Enferm., v.21, n.2, p.363-370, 2012. doi: 10.1590/S010407072012000200014

BALDIN, N.; MUNHOZ, E.M.B. Snowball (bola de neve): uma técnica metodológica para pesquisa em educação ambiental comunitária. In: CONGRESSO NACIONAL DE EDUCAÇÃOEDURECE, 10, ; 2011, Curitiba, Anais... Curitiba: PUCPR, 2011. p.329-341,

BARROS, N.F. A construção da medicina integrativa: um desafio para o campo da saúde. São Paulo: Hucitec, 2006.

BRASIL. Ministério da Saúde. Secretaria de Atenção à Saúde. Área da Saúde da Criança. Atenção integrada às doenças prevalentes na infância: curso de capacitação. Módulo 4 . Brasília: MS, 2003.

BRASIL. Ministério da Saúde. Portaria Interministerial no 2.960, de 9 de dezembro de 2008. Dispõe sobre a aprovação o Programa Nacional de Plantas Medicinais e Fitoterápicos e cria o Comitê Nacional de Plantas Medicinais e Fitoterápicos. Diário Oficial da União, 2008.

BRASIL. Ministério da Saúde. Secretaria de Atenção à Saúde. Departamento de Atenção Básica. Política nacional de práticas integrativas e complementares no SUS: atitude de ampliação de acesso. Brasília: MS, 2015.

CHARCHAT-FICHMAN, H. et al. Declínio da capacidade cognitiva durante o envelhecimento. Rev. Bras. Psiquiatr., v.27, n.1, p.79-82, 2005. doi: 10.1590/S1516-44462005000100017

CHAVES, E.M.F.; BARROS, R.F.M. Diversidade e uso de recursos medicinais do carrasco na APA da Serra da Ibiapaba, Piauí, Nordeste do Brasil. Rev. Bras. Plantas Med., v.14, n.3, p.476-486, 2012. doi: 10.1590/S1516-05722012000300009

CONTATORE, O. A. et al. Uso, cuidado e política das práticas integrativas e complementares na Atenção Primária à Saúde. Ciênc. Saúde Coletiva., v.20, n.10, p.3263-3273, 2015. doi: 10.1590/1413-812320152010.00312015

FEIJÓ, E.V.R.S. et al. Levantamento preliminar sobre plantas medicinais utilizadas no bairro Salobrinho no município de Ilhéus, Bahia. Rev. Bras. Plantas Med., v.15, n.4, p. 595-604, 2013. doi: 10.1590/S1516-05722013000400017

FERREIRA, F.M.C.; LOURENÇO, F.J.; BALIZA, D.P. Ethnobotanical survey of medicinal plants in maroon community Carreiros, Mercês - Minas Gerais. Rev. Verde, v.9, n.3, p.205212, 2014.

FISCHER, M.L.; PALODETO, M.F.T.; SANTOS, E.C. Uso de animais como zooterápicos: uma questão bioética. Histor. Ciênc. Saude., v.25, n.1, 2018. doi: 10.1590/s0104-59702018000100013

FREITAS, A.V.L. et al. Plantas medicinais: um estudo etnobotânico nos quintais do Sítio Cruz, São Miguel, Rio Grande do Norte, Brasil. Rev. Bras. Bioci., v.10, n.1, p.48-59, 2012.

GIRALDI, M.; HANAZAKI, N. Uso e conhecimento tradicional de plantas medicinais no Sertão do Ribeirão, Florianópolis, SC, Brasil. Acta Bot. Bras., v.24, n.2, p.395-406, 2010. doi: 10.1590/ S0102-33062010000200010

HAVERROTH, M. Etnobiologia e saúde de povos indígenas. Recife: NUPEEA, 2013.

HEISLER, E.V. et al. Saber popular sobre a utilização da planta Anredera cordifolia (folha gorda). Texto Contexto Enferm., v.21, n.4, p.937-944, 2012. doi: 10.1590/S0104-07072012000400026

LANGDON, E.J. Os diálogos da antropologia com a saúde: contribuições para as políticas públicas. Ciênc. Saúde Coletiva, v.19, n.4, p.1019-1029, 2014. doi: 10.1590/141381232014194.22302013

LIMA, A.R.A. et al. Medicinal plants used by octogenarians and nonagenarians from a small village in Rio Grande/RS, Brazil. $J$. Nurs. UFPE, v.5, n.6, p.1329-336, 2011.

LOPES, L.C. et al. Brazilian medicinal plants to treat upper respiratory tract and bronchial illness: systematic review and meta-analyses-study protocol. BMJ., v.4, n.7, p.1-6, 2014.

MARCHESE, J.A. et al. Medicinal plants used by "Passo da Ilha" rural community in the city of Pato Branco, southern Brazil. 
An. Acad. Bras. Ciênc., v.81, n.4, p.692-700, 2009. doi: 10.1590/ S0001-37652009000400008

OLIVEIRA, F.C.S.; BARROS, R.F.M.; MOITA NETO, J.M. Plantas medicinais utilizadas em comunidades rurais de Oeiras, semiárido piauiense. Rev. Bras. Plantas Med., v.12, n.3, p.282301, 2010. doi: 10.1590/S1516-05722010000300006

PIMENTEL-GOMES, F. Curso de estatística experimental. Piracicaba: Nobel, 1990.

RODRIGUES, A.P.; ANDRADE, L.H.C. Levantamento etnobotânico das plantas medicinais utilizadas pela comunidade de Inhamã, Pernambuco, Nordeste do Brasil. Rev. Bras. Plantas Med., v.16, n.3, p.721-730, 2014. doi: 10.1590/1983-084x/08_159

SANTOS, M.E.A.; QUINTAO, N.T.; ALMEIDA, R.X. Avaliação dos marcos do desenvolvimento infantil segundo a estratégia da atenção integrada às doenças prevalentes na infância. Esc. Anna Nery., v.14, n.3, p.591-591, 2010. doi: 10.1590/S141481452010000300022

SAPAROLLI, E.C.L.; ADAMI, N.P. Avaliação da qualidade da consulta de enfermagem à criança no Programa de Saúde da Família. Acta Paul. Enferm., v.20, n.1, p.55-61, 2007. doi: $10.1590 / \mathrm{S} 0103-21002007000100010$

SILVA, A.A. et al. OS Agentes da cura na medicina rústica brasileira. Rev. Int. Est. Saúde., v.6, n.1, p. 72-84, 2017.

SOUSA, I.M.C.; HORTALE, V.A.; BODSTEIN, R.C.A. Medicina tradicional complementar e integrativa: desafios para construir um modelo de avaliação do cuidado. Ciênc. Saúde Coletiva., v.23, n.10, p. 3403-3412, 2018. doi: 10.1590/1413812320182310.23792016

SOUSA, I.M.C.; TESSER, C.D. Medicina tradicional e complementar no Brasil: inserção no sistema único de saúde e integração com a atenção primária. Cad. Saúde Pública., v.33, n.1, p.1-15, 2017. doi: 10.1590/0102-311X00150215

SOUZA, A.D.Z. et al. Plantas medicinais utilizadas na saúde da criança. Rev. Enferm. Global., n.24, p.53-59, 2011.

SOUZA, C.M.P. et al. Utilização de plantas medicinais com atividade antimicrobiana por usuários do serviço público de saúde em Campina Grande - Paraíba. Rev. Bras. Plantas Med., v.15, n.2, p.188-193,2013. doi: 10.1590/S1516-05722013000200004

VINUTO, J. A amostragem em bola de neve na pesquisa qualitativa: um debate em aberto. Rev. Temáticas, v.22, n.44, p.203-220, 2014.

ZENI, A.L.B. et al. Utilização de plantas medicinais como remédio caseiro na Atenção Primária em Blumenau, Santa Catarina, Brasil. Ciênc. Saúde Coletiva, v.22, n.8, p.2703-2712, 2017. doi: 10.1590/1413-81232017228.18892015

ZUCCHI, M.R. et al. Levantamento etnobotânico de plantas medicinais na cidade de Ipameri - GO. Rev. Bras. Plantas Med., v.15, n.2, p.273-279, 2013. doi: 10.1590/S151605722013000200016 